\title{
Contribution à l'étude des spectres composites
}

\author{
VIII. HD 174016-7, une étoile Ap associée à une géante $G^{\star}$
}

\section{Contribution to the study of composite spectra VIII. HD 174016-7, an Ap star with a giant G}

\author{
N. Ginestet ${ }^{1}$, R.F. Griffin ${ }^{2}$, J.M. Carquillat ${ }^{1}$, et S. Udry ${ }^{3}$ \\ 1 Observatoire Midi-Pyrénées, Laboratoire d'astrophysique (UMR 5572), 14 avenue Édouard Belin, F-31400 Toulouse, France \\ 2 The Observatories, Madingley Road, Cambridge CB3 0HA, UK \\ 3 Observatoire de Genève, CH-1290 Sauverny, Suisse
}

Reçu le 23 juillet ; accepté le 27 août 1999

\begin{abstract}
HD 174016-7, listed by Hynek (1938) as a star having a composite spectrum, was on our observing programmes of such objects carried out both at the Cambridge Observatories and at the Observatoire MidiPyrénées. Most of the observations were made with the CORAVEL spectrovelocimeter of the Swiss telescope at the Observatoire de Haute-Provence. We find that this star is a long-period spectroscopic binary with two correlation dips; we obtain the following orbital elements: $P=3097.9$ days; $T=\mathrm{JD} 2450605.2 ; \omega=204.8 ; e=$ $0.600 ; K_{1}=12.95 \mathrm{~km} \mathrm{~s}^{-1} ; K_{2}=15.14 \mathrm{~km} \mathrm{~s}^{-1} ; V_{0}=$ $-1.65 \mathrm{~km} \mathrm{~s}^{-1} ; a_{1} \sin i=441.1 \mathrm{Gm} ; a_{2} \sin i=516.0 \mathrm{Gm}$; $M_{1} \sin ^{3} i=1.967 M_{\odot} ; M_{2} \sin ^{3} i=1.681 M_{\odot}$. The primary is a giant star of spectral type near G6III, and the hot dwarf secondary is found to be a peculiar A star of type A0p Sr, Cr, Eu, Si; so HD 174016-7 is, to our knowledge, the second discovered composite-spectrum binary with a Ap-type hot component. A confrontation with Hipparcos data suggests $M_{v_{1}}=0$ and $m_{v}=0.6 \mathrm{mag}$. On the basis of very accurate masses of main sequence stars by Andersen (1991), we estimate the mass, $M_{1}=2.8 M_{\odot}$, of the giant primary, the orbital inclination, $i=63^{\circ}$, and the mean linear separation of the components, $a=7.2$ AU. The evolutionary status of the system is discussed using Schaller et al. (1992) $M_{\mathrm{bol}} / T_{\text {eff }}$ diagram for stars of solar metallicity. Theoretical masses suggested by this diagram confirm the proposed model.
\end{abstract}

Key words: stars: individual: HD 174016-7 — binaries: spectroscopic; symbiotic — stars: fundamental parameters

\footnotetext{
* Étude effectuée à partir d'observations faites aux Observatoires de Haute-Provence et de Cambridge.
}

\section{Introduction}

HD 174016-7 (= BD + 61 $1771=$ HIP 91921), étoile de 8ème magnitude dans la constellation du Dragon, figurait dans la liste des étoiles à spectre composite répertoriées par Hynek (1938), principale source de nos programmes d'observations systématiques en vitesse radiale de ces objets, afin d'en étudier la binarité (Griffin 1986; Carquillat et al. 1988).

La nature "spectre composite" de cette étoile était, en fait, déjà mise en évidence dans le Henry Draper Catalogue (Cannon \& Pickering 1922) : double numérotation et types spectraux F5, A2, attribués respectivement aux deux composantes. Hynek (1938) a simplement reporté la classification du catalogue HD, mais par la suite HD 174016-7 est citée dans plusieurs articles traitant de problèmes de classifications :

- un travail de Olsen (1980) porte sur des classifications d'étoiles suspectées particulières d'après leurs indices dans la photométrie de Strömgren. Dans cette investigation, Olsen propose pour HD 174016-7 les possibilités suivantes : 1) spectre composite (hypothèse la plus probable), 2) Fm/p, 3) sgF5sl (sl pour "strong metallic lines") ;

- un travail de Abt (1984) a pour but de tester par des observations spectrographiques (plaques Kodak IIa-O, dispersion $39 \AA / \mathrm{mm}$ ) les classifications suggérées par Olsen. Ainsi, d'après Abt, HD 174016-7 serait une étoile Am, avec les classifications A1, F3, F5 respectivement pour la raie $\mathrm{K}$ de $\mathrm{CaII}$, les raies d'hydrogène et les raies métalliques : aussi figure-t-elle dans le catalogue des étoiles Am de Hauck (1986) ;

- des travaux basés sur des observations effectuées dans le proche infrarouge montrent, sans ambiguïté, la nature composite du spectre de HD 174016-7 (Ginestet et al. 
Tableau 1. Vitesses radiales de HD 174016-7. VR1, $(\mathrm{O}-\mathrm{C})_{1}$ : vitesses radiales observées et résidus pour la primaire ; VR2, $(\mathrm{O}-\mathrm{C})_{2}$ : mêmes quantités pour la secondaire. Colonne $9: \mathrm{OHP}=\mathrm{CORAVEL} ; \mathrm{C} 1=$ ancien spectromètre de Cambridge ; $\mathrm{C} 2=$ nouveau spectromètre de Cambridge

\begin{tabular}{|c|c|c|c|c|c|c|c|c|}
\hline $\mathrm{N}^{\circ}$ & Date & $\begin{array}{r}\text { Date JJ } \\
2400000+\end{array}$ & Phase & $\begin{array}{c}\text { VR1 } \\
\mathbf{k m ~ s}^{-1}\end{array}$ & $\begin{array}{l}(\mathrm{O}-\mathrm{C})_{1} \\
\mathbf{k m ~ s}^{-1}\end{array}$ & $\begin{array}{c}\text { VR2 } \\
\mathbf{k m ~ s}^{-1}\end{array}$ & $\begin{array}{l}(\mathrm{O}-\mathrm{C})_{2} \\
\mathrm{~km} \mathrm{~s}^{-1}\end{array}$ & Obs. \\
\hline 1 & 13.09 .1981 & 44861,316 & 0,146 & $+3,5$ & $+1,1$ & $-5,7$ & $+0,7$ & OHP \\
\hline 2 & $15 . " \quad$ & 44863,308 & 0,146 & $+2,7$ & $+0,3$ & $-6,7$ & $-0,3$ & OHP \\
\hline 3 & $16 . \quad "$ & 44864,297 & 0,147 & $+3,3$ & $+0,9$ & $-6,4$ & $+0,0$ & OHP \\
\hline 4 & 21.08 .1982 & 45203,360 & 0,256 & $+4,5$ & $+0,3$ & $-9,2$ & $-0,7$ & $\mathrm{OHP}$ \\
\hline 5 & 22. " " & 45204.365 & 0,257 & $+4,2$ & $+0,0$ & $-7,8$ & $+0,6$ & OHP \\
\hline 6 & $25 . \quad "$ & 45207,325 & 0,258 & $+3,1$ & $-1,1$ & $-10,4$ & $-1,9$ & OHP \\
\hline 7 & 19.09 .1983 & 45597,302 & 0,383 & $+3,7$ & $-0,3$ & $-8,0$ & $+0,2$ & OHP \\
\hline 8 & 13.06 .1985 & 46229,538 & 0,588 & $+1,8$ & $-0,1$ & $-5,8$ & $+0,0$ & OHP \\
\hline 9 & 04.05 .1988 & 47285,629 & 0,928 & $-13,8$ & $+0,3$ & $+13,7$ & $+0,8$ & OHP \\
\hline 10 & $06 . \quad "$ & 47287,589 & 0,929 & $-14,4$ & $-0,2$ & $+12,3$ & $-0,7$ & OHP \\
\hline 11 & 14.06 .1988 & 47326,500 & 0,942 & $-15,9$ & $+0,2$ & & & $\mathrm{C} 1$ \\
\hline 12 & 24.10 .1988 & 47459,347 & 0,984 & $-21,0$ & $+0,6$ & $+22,9$ & $+1,2$ & OHP \\
\hline 13 & 27. " & 47462,328 & 0,985 & $-21,3$ & $+0,3$ & $+21,8$ & $+0,1$ & OHP \\
\hline 14 & 06.11 .1988 & 47472,252 & 0,989 & $-21,7$ & $-0,1$ & $+21,1$ & $-0,6$ & OHP \\
\hline 15 & 30. " " & 47496,238 & 0,996 & $-21,1$ & $-0,4$ & & & $\mathrm{Cl}$ \\
\hline 16 & 16.03 .1989 & 47601,688 & 0,030 & $-12,4$ & $-0,2$ & $+9,7$ & $-0,9$ & OHP \\
\hline 17 & 23. " " & 47608.671 & 0,033 & $-11,8$ & $-0,3$ & $+9,6$ & $-0,3$ & OHP \\
\hline 18 & 25. " & 47610,667 & 0,033 & $-11,4$ & $-0,1$ & $+10,3$ & $+0,6$ & OHP \\
\hline 19 & 29.04 .1989 & 47645,646 & 0,045 & $-8,4$ & $-0,1$ & $+5,3$ & $-0,8$ & OHP \\
\hline 20 & 27.01 .1990 & 47918,723 & 0,133 & $+2,0$ & $+0,1$ & $-4,1$ & $+1,7$ & OHP \\
\hline 21 & 16.03 .1990 & 47966,708 & 0,148 & $+2,1$ & $-0,4$ & $-5,9$ & $+0,6$ & OHP \\
\hline 22 & 24.08 .1990 & 48128,386 & 0,200 & $+3,1$ & $-0,6$ & $-8,5$ & $-0,6$ & OHP \\
\hline 23 & $29 . " 1$ & 48133,378 & 0,202 & $+3,2$ & $-0,5$ & $-6,0$ & $+1,9$ & OHP \\
\hline 24 & 29.01 .1991 & 48285,732 & 0,251 & $+4,2$ & $+0,1$ & $-7,3$ & $+1,1$ & OHP \\
\hline 25 & 29.10 .1991 & 48559,289 & 0,340 & $+4,1$ & $-0,1$ & $-7,8$ & $+0,7$ & OHP \\
\hline 26 & 18.12.1991 & 48609,242 & 0,356 & $+4,2$ & $+0,1$ & $-7,3$ & $+1,1$ & OHP \\
\hline 27 & 30.04 .1992 & 48742,617 & 0,399 & $+4,3$ & $+0,4$ & $-5,7$ & $+2,4$ & OHP \\
\hline 28 & 21.06 .1992 & 48794,543 & 0,416 & $+3,6$ & $-0,2$ & $-7,5$ & $+0,5$ & OHP \\
\hline 29 & 13.08 .1992 & 48848,438 & 0,433 & $+3,5$ & $-0,2$ & $-7,1$ & $+0,8$ & OHP \\
\hline 30 & 20.12 .1992 & 48977,233 & 0,474 & $+3,2$ & $-0,1$ & $-6,6$ & $+0,8$ & $\mathrm{OHP}$ \\
\hline 31 & 18.02 .1993 & 49036,709 & 0,494 & $+3,2$ & $+0,1$ & $-6,5$ & $+0,7$ & $\mathrm{OHP}$ \\
\hline 32 & 03.06 .1993 & 49141,528 & 0,528 & $+2,7$ & $-0,0$ & $-6,3$ & $+0,5$ & OHP \\
\hline 33 & $05 . \quad "$ & 49143,528 & 0,528 & $+2,5$ & $-0,2$ & $-5,3$ & $+1,5$ & OHP \\
\hline 34 & $06 . \quad "$ & 49144,547 & 0,528 & $+2,8$ & $+0,1$ & $-6,2$ & $+0,6$ & OHP \\
\hline 35 & $08 . \quad "$ & 49146,520 & 0,529 & $+2,0$ & $-0,7$ & $-6,6$ & $+0,2$ & OHP \\
\hline 36 & $08 . \quad "$ & 49146,612 & 0,529 & $+2,1$ & $-0,6$ & $-4,9$ & $+1,9$ & OHP \\
\hline 37 & 09.07 .1993 & 49177,500 & 0,539 & $+2,6$ & $+0,0$ & $-4,3$ & $+2,3$ & OHP \\
\hline 38 & 25.11 .1993 & 49317,281 & 0,584 & $+1,5$ & $-0,5$ & $-5,1$ & $+0,8$ & OHP \\
\hline 39 & 03.12 .1993 & 49325,273 & 0,587 & $+2,0$ & $+0,1$ & $-4,7$ & $+1,1$ & OHP \\
\hline 40 & 04.01 .1994 & 49357,246 & 0,597 & $+2,0$ & $+0,2$ & $-4,3$ & $+1,4$ & OHP \\
\hline 41 & 17.03 .1994 & 49428,671 & 0,620 & $+1,4$ & $+0,0$ & $-4,0$ & $+1,2$ & OHP \\
\hline 42 & 03.05 .1994 & 49475,621 & 0,635 & $+1,2$ & $+0,1$ & $-4,2$ & $+0,7$ & $\mathrm{OHP}$ \\
\hline 43 & 05.08 .1994 & 49570.475 & 0,666 & $+0,1$ & $-0,4$ & $-3,0$ & $+1,2$ & OHP \\
\hline 44 & 05.06 .1995 & 49873,579 & 0,764 & $-1,9$ & $+0,2$ & $-0,2$ & $+0,9$ & OHP \\
\hline 45 & 14.02 .1996 & 50127,716 & 0,846 & $-6,1$ & $-0,1$ & $+2,6$ & $-0,9$ & OHP \\
\hline 46 & 04.04 .1996 & 50177,639 & 0,862 & $-6,8$ & $+0,3$ & $+6,5$ & $+1,7$ & $\mathrm{OHP}$ \\
\hline 47 & $20 . \quad "$ & 50193,639 & 0,867 & $-7,9$ & $-0,4$ & $+6,0$ & $+0,8$ & OHP \\
\hline 48 & 26. " & 50199,642 & 0,869 & $-7,4$ & $+0,3$ & $+7,0$ & $+1,6$ & $\mathrm{OHP}$ \\
\hline 49 & 29.08 .1996 & 50325,413 & 0,910 & $-11,4$ & $+0,2$ & $+11,1$ & $+1,1$ & $\mathrm{OHP}$ \\
\hline 50 & 02.09 .1996 & 50329,485 & 0,911 & $-11,5$ & $+0,3$ & $+11,6$ & $+1,4$ & OHP \\
\hline 51 & 17.10 .1996 & 50374.288 & 0.925 & $-13,9$ & $-0,3$ & $+12,3$ & $-0,1$ & $\mathrm{OHP}$ \\
\hline
\end{tabular}


Tableau 1. continued

\begin{tabular}{|c|c|c|c|c|c|c|c|c|}
\hline 52 & 26.11 .1996 & 50414,279 & 0,938 & $-15,7$ & $-0,1$ & $+15,2$ & $+0,6$ & OHP \\
\hline 53 & 30. " & 50418,331 & 0,940 & $-15,6$ & $+0,2$ & & & OHP \\
\hline 54 & 03.12 .1996 & 50421,304 & 0,941 & $-16,6$ & $-0,7$ & $+14,8$ & $-0,2$ & OHP \\
\hline 55 & 17. " & 50435,241 & 0,945 & $-17,2$ & $-0,6$ & $+14,7$ & $-1,2$ & OHP \\
\hline 56 & 26.01 .1997 & 50474,720 & 0,958 & $-19,1$ & $-0,4$ & $+19,0$ & $+0,7$ & OHP \\
\hline 57 & 29. " & 50477,751 & 0,959 & $-19,1$ & $-0,2$ & $+18,5$ & $+0,0$ & OHP \\
\hline 58 & 01.03 .1997 & 50508,680 & 0,969 & $-20,5$ & $-0,1$ & $+20,5$ & $+0,3$ & $\mathrm{C} 2$ \\
\hline 59 & $29 . \quad "$ & 50536,680 & 0,978 & $-21,1$ & $+0,2$ & $+22,0$ & $+0,6$ & $\mathrm{C} 2$ \\
\hline 60 & 11.04 .1997 & 50549,650 & 0,982 & $-21,6$ & $-0,0$ & $+22,1$ & $+0,4$ & $\mathrm{C} 2$ \\
\hline 61 & 25. " & 50563,610 & 0,987 & $-21,4$ & $+0,2$ & $+21,3$ & $-0,4$ & OHP \\
\hline 62 & 07.05 .1997 & 50575,690 & 0,990 & $-21,7$ & $-0,2$ & $+22,3$ & $+0,7$ & $\mathrm{C} 2$ \\
\hline 63 & 10.06 .1997 & 50609,610 & 0,001 & $-20,2$ & $+0,0$ & $+16,4$ & $-3,6$ & OHP \\
\hline 64 & $16 . "$ & 50615,583 & 0,003 & $-20,0$ & $-0,2$ & $+18,9$ & $-0,7$ & OHP \\
\hline 65 & 21. " & 50620,551 & 0,005 & $-19,5$ & $-0,0$ & $+19,5$ & $+0,3$ & $\mathrm{C} 2$ \\
\hline 66 & 07.07 .1997 & 50636,500 & 0,010 & $-18,2$ & $+0,0$ & $+19,0$ & $+1,3$ & $\mathrm{C} 2$ \\
\hline 67 & 17.08 .1997 & 50678,452 & 0,024 & $-14,0$ & $+0,2$ & $+13,9$ & $+0,8$ & $\mathrm{OHP}$ \\
\hline 68 & 09.09 .1997 & 50701,361 & 0,031 & $-12,4$ & $-0,4$ & $+10,3$ & $-0,1$ & OHP \\
\hline 69 & $14 . " 1$ & 50706,366 & 0,033 & $-11,7$ & $-0,2$ & $+10,1$ & $+0,2$ & OHP \\
\hline 70 & 23.10 .1997 & 50745,292 & 0.045 & -8.0 & $+0,2$ & $+7,6$ & +1.6 & OHP \\
\hline 71 & 22.12 .1997 & 50805,290 & 0,065 & $-4,2$ & $+0,1$ & $+1,6$ & $+0,2$ & OHP \\
\hline 72 & 20.10 .1998 & 51107,329 & 0,162 & $+2,8$ & $-0,1$ & $-5,3$ & $+1,7$ & OHP \\
\hline 73 & 21. " & 51108,370 & 0,162 & $+2,2$ & $-0,7$ & $-6,6$ & $+0,4$ & $\mathrm{OHP}$ \\
\hline 74 & 22. & 51109,298 & 0,163 & $+2,5$ & $-0,4$ & $-6,6$ & $+0,4$ & OHP \\
\hline
\end{tabular}

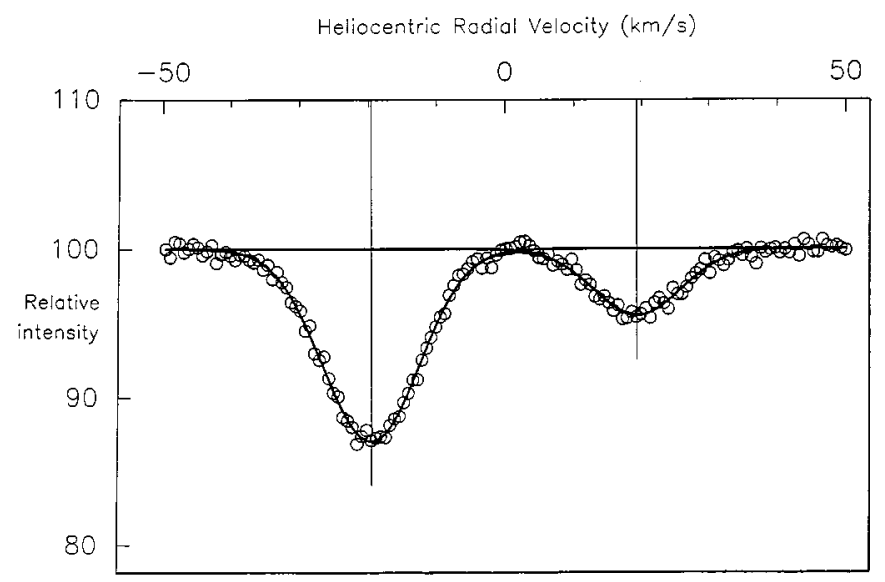

Fig. 1. Traces de corrélation des composantes de HD 174016-7 au voisinage du passage au périastre obtenues par R.F.G. avec le nouveau spectromètre de Cambridge

1997; 1999). Deux remarques méritent ici d'être formulées. La première concerne la confusion entre étoile Am et étoile à spectre composite : dans la plupart des cas où il y a eu confusion, il s'agit d'étoiles qui étaient classées composites dans le HD et qui, par la suite, se sont révélées être de type Am, telles, par exemple, HD 41724-5 (Carquillat et al. 1988), HD 51565-6 (Griffin et al. 1997), HD 66068-9 (Carquillat et al. 1994), HD 83270-1 (Ginestet et al. 1991) et HD 177390-1 (Griffin 1988; Carquillat et al. 1988) ; les raisons de cette confusion sont clairement rappelées dans Griffin et al. (1997). Par contre, dans le cas présent, nos récentes classifications ont confirmé, sans aucun doute pos- sible, le caractère composite de HD 174016-7, et non le caractère Am indiqué par Abt. La deuxième remarque est pour souligner le fait que, dans les anciennes classifications d'étoiles à spectre composite, les composantes froides sont, quasi systématiquement, classées trop "chaudes" à cause d'un biais dû à l'enchevêtrement des spectres des composantes dans les zones spectrales du bleu et du visible, ce qui explique l'écart entre F5 et G8. Dans la Sect. 3 nous discuterons des classifications que nous proposons pour les composante de ce système.

Le Centre de Données de Strasbourg (CDS), quant à lui, a retenu pour cet objet une classification spectrale unique, F5.

Enfin, concernant la vitesse radiale de HD 174016-7, rien à ce jour, à notre connaissance, n'avait été publié ; nos observations ont mis en évidence la nature binaire spectroscopique de cette étoile, avec une période orbitale voisine de 8,5 années.

\section{Observations et détermination des éléments orbitaux}

HD 174016-7 est une binaire spectroscopique à deux spectres (BS2), et nous avons déterminé ses éléments orbitaux grâce à 74 observations en vitesses radiales (VR) du système : $74 \mathrm{VR}$ ont été obtenues pour la composante froide, la primaire, et 71 pour la composante chaude, la secondaire ; ces VR (Tableau 1) sont données dans le système international des standards de l'UAI.

La plupart de ces observations ont été effectuées à l'Observatoire de Haute-Provence (OHP) au moyen du 


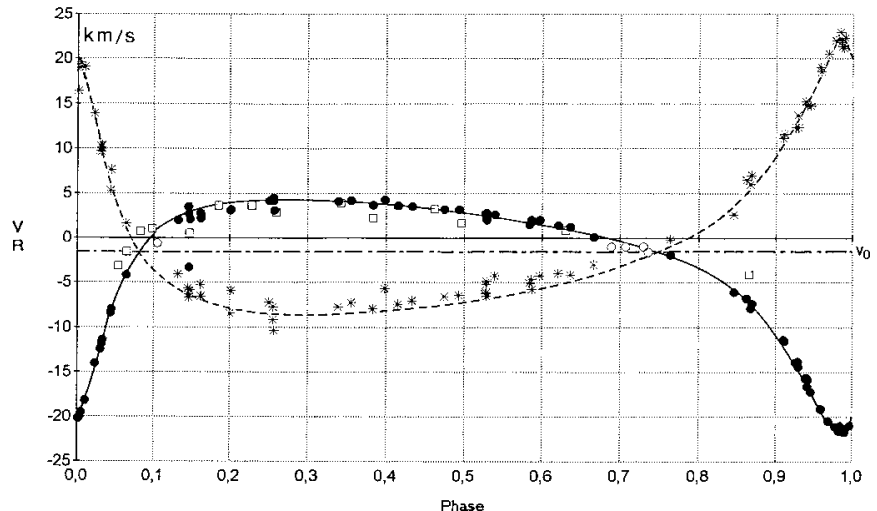

Fig. 2. Courbes de vitesse radiale des deux composantes de HD 174016-7. Cercles pleins : étoile primaire ; astérisques : étoile secondaire ; composantes non résolues en VR : cercles vides (OHP), carrés vides (Cambridge, Victoria). L'origine des phases est l'époque de passage au périastre

spectrovélocimètre CORAVEL (Baranne et al. 1979) monté sur le télescope suisse de $1 \mathrm{~m}$, à l'exception de huit VR obtenues à l' Observatoire de Cambridge. Ces observations s'étendent sur 16 années (1982 à 1998), soit près de deux cycles orbitaux. La réduction des VR obtenues à CORAVEL a été effectuée à l'Observatoire de Genève par l'un d'entre nous (S.U.) au moyen d'un programme spécifique de déconvolution des traces de corrélation (Duquennoy 1987). L'erreur standard moyenne interne sur les VR est de $0,56 \mathrm{~km} \mathrm{~s}^{-1}$ pour la primaire et $1,04 \mathrm{~km} \mathrm{~s}^{-1}$ pour la secondaire, pour les observations OHP. Les huit VR mesurées à Cambridge ont été obtenues avec deux appareils différents, conçus par R.F.G. : deux avec l'ancien spectromètre photoélectrique (Griffin 1967), et six avec un nouveau spectromètre, de type Coravel ; afin de rendre ces VR compatibles avec les VR CORAVEL, une correction de $+0,8 \mathrm{~km} \mathrm{~s}^{-1}$ a été appliquée à celles mesurées au moyen de l'ancien spectromètre, tandis que celles provenant du nouveau spectromètre devaient être diminuées de $1,5 \mathrm{~km} \mathrm{~s}^{-1}$. À la Fig. 1, nous donnons un enregistrement des traces de corrélation des deux composantes obtenues avec le nouveau spectromètre de Cambridge, pour une phase où ces composantes sont proches de leur séparation maximum en VR.

Les éléments orbitaux définitifs, donnés ci-après, ont été calculés avec notre programme BS2 (Nadal et al. 1979). Les pondérations adoptées pour effectuer ce calcul sont les suivantes : pour les VR obtenues à l'OHP, 1 pour la primaire, $1 / 8$ pour la secondaire ; pour celles mesurées avec le nouveau spectromètre de Cambridge, 2 pour la primaire, $1 / 4$ pour la secondaire ; et enfin $1 / 3$ pour les deux mesures provenant de l' ancien spectromètre de Cambridge. Ces valeurs ont été retenues après analyse des résidus $\mathrm{O}-\mathrm{C}$ donnés par un calcul préliminaire. Nous obtenons :

$P=3097,9 \pm 2,2$ jours

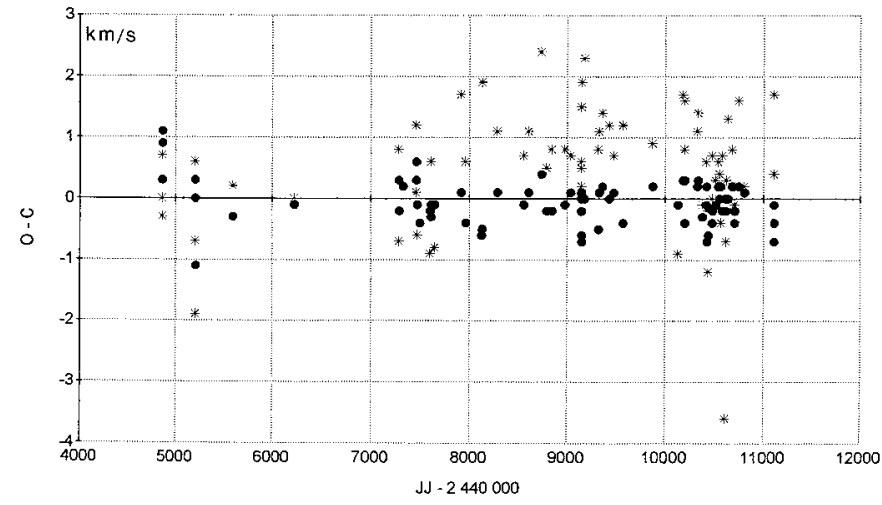

Fig. 3. Résidus $\mathrm{O}-\mathrm{C}$ en fonction du temps (Jour Julien). Cercles pleins : composante primaire ; astérisques : composante sercondaire

$T=$ JJ $2450605,2 \pm 2,0$
$\omega=204,8^{\circ} \pm 0,6^{\circ}$
$e=0,600 \pm 0,003$
$K_{1}=12,95 \pm 0,06 \mathrm{~km} \mathrm{~s}^{-1}$
$K_{2}=15,14 \pm 0,15 \mathrm{~km} \mathrm{~s}^{-1}$
$V_{0}=-1,65 \pm 0,05$
$a_{1} \sin i=441,1 \pm 2,4 \mathrm{Gm}$
$a_{2} \sin i=516,0 \pm 5,3 \mathrm{Gm}$
$M_{1} \sin ^{3} i=1,967 \pm 0,045 M_{\odot}$
$M_{2} \sin ^{3} i=1,681 \pm 0,027 M_{\odot}$
$\sigma(\mathrm{O}-\mathrm{C})=0,36($ pour une $\mathrm{VR}$ de poids unité).

Les courbes de VR des deux composantes sont présentées à la Fig. 2. Sur cette figure ont été reportées aussi les observations correspondant à des traces de corrélation "blendes" pour lesquelles les VR mesurées étaient susceptibles d'être faussées par la présence du pic, non résolu, de la secondaire : 5 observations effectuées à l'OHP, 13 observations à Cambridge (ancien spectromètre) et une au Dominion Astrophysical Observatory (Victoria) sont concernées ; bien évidemment ces 19 observations n'ont pas été utilisées pour le calcul des éléments orbitaux.

Les écarts O-C entre les VR observées et les VR calculées avec ces éléments sont donnés en Table 1, et en Fig. 3 ils sont représentés en fonction du temps. Nous constatons que les résidus $(\mathrm{O}-\mathrm{C})_{1}$ de la primaire sont faibles et semblent répartis à peu près au hasard autour de la valeur zéro, ce qui n'est pas le cas pour ceux, $(\mathrm{O}-\mathrm{C})_{2}$, de la secondaire. La dispersion plus grande de ces derniers pourrait être attribuée à la nature Ap de cette composante, par suite de variations de VR intrinsèques dues à des inhomogéniétés d'abondances à sa surface, mais il apparaît surtout que les quantités $(\mathrm{O}-\mathrm{C})_{2}$ sont systématiquement positives pour les phases où les traces de corrélation des composantes sont les plus imbriquées ; cette anomalie pourrait ainsi résulter d'un effet résiduel dû à la proximité, en vitesse radiale, de la trace de la composante primaire pour les phases situées de part et d'autre du passage à l'apoastre. 


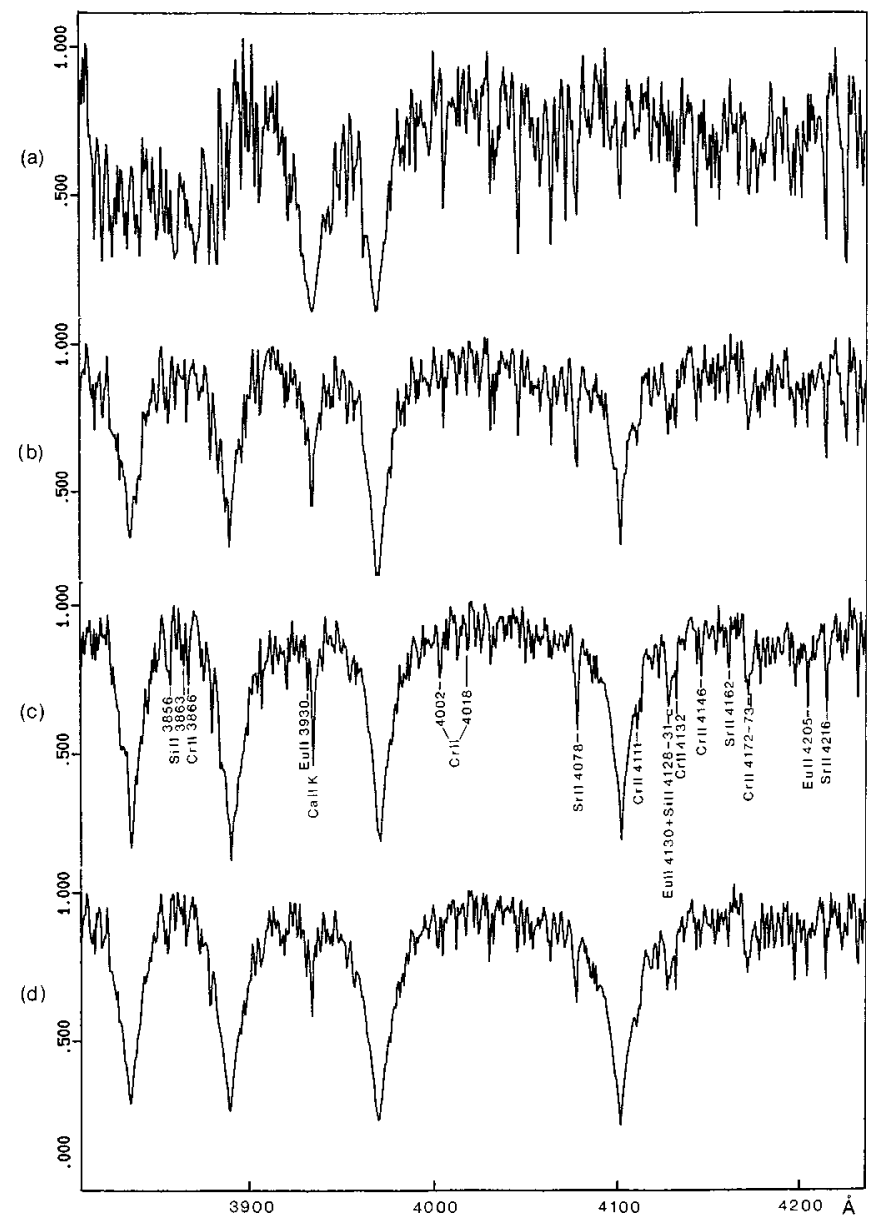

Fig. 4. a) spectre dans la région du bleu - proche UV de $\varepsilon$ Vir, standard MK de type G8III ; b) spectre de HD 174016-7 ; c) spectre du compagnon chaud (A0p) obtenu par soustraction du spectre de $\varepsilon$ Vir (G8III) de celui de HD 174016-7 ; d) spectre de l'étoile HD 71866 de type A0p

\section{Classifications spectrales}

Dans l'introduction nous avons signalé qu'un travail de classifications dans le proche IR d'étoiles à spectre composite confirmait le caractère composite de HD 174016. La première investigation (Ginestet et al. 1997), effectuée avec des spectres CCD obtenus au moyen du spectrographe CARELEC du Télescope $193 \mathrm{~cm}$ de l'OHP, conduisait à attribuer à la composante froide la classification G8III-IV : le domaine spectral était 8370-8780 § et la dispersion était de $33 \AA / \mathrm{mm}$. A la suite d'une étude complémentaire (Ginestet et al. 1999), toujours dans le proche IR mais avec un domaine spectral plus étendu $(500 \AA \mathrm{au}$ lieu de $400 \AA$ ) et un autre instrument de l'OHP (spectrographe AURELIE -Télescope $152 \mathrm{~cm}$ ) nous avons révisé légèrement notre classification et retenu le type spectral G6III. Dans la suite de cette analyse, nous verrons que les données d'Hipparcos confirment que la composante évoluée de HD 174016-7 doit en effet être une étoile géante.

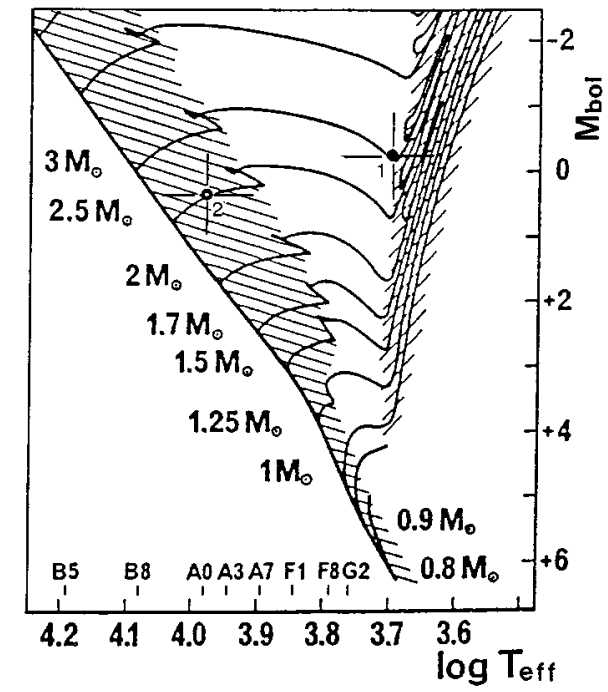

Fig. 5. Position des composantes de HD 174016-7 dans le diagramme HR théorique $M_{\mathrm{bol}} / \log T_{\text {eff }}$ de Schaller et al. (1992). Les zones hachurées correspondent aux phases de combustion nucléaire lente. Cercle plein : composante primaire (1) ; cercle vide : composante secondaire (2)

Des observations, récemment effectuées dans le domaine spectral du bleu - proche UV $(3800-4200 \AA)$ au moyen du spectrographe AURELIE $(R=7000$; $16 \AA / \mathrm{mm}$ ), nous permettent de préciser également la nature de la composante chaude. Le fait que cette dernière donne un pic de corrélation à CORAVEL laissait déjà supposer qu'elle devait être une étoile chimiquement particulière de type Am ou Ap, car lorsque les composantes chaudes sont des étoiles normales de types B ou A elles ne peuvent être détectées avec cet instrument conçu pour la mesure des étoiles froides.

L'examen des spectres dans ce domaine $3800-4200 \AA$ nous a permis de détecter la présence d'une étoile Ap de type Sr, Cr, Eu, Si : en effet les raies de SrII 4078 et 4216 sont fortement dominantes, on trouve également de nombreuses et fortes raies de CrII (3866, 4002, 4012, 4018, 4111, 4132, 4146, 4172) ainsi que des raies de EuII (3930, $4130,4205)$ et des raies de $\operatorname{SiII}(3856,4128,4131)$; le profil de la raie $\mathrm{K}$ de Ca II indique une étoile A des premiers types spectraux, A0 ou A1. Cette classification est d'ailleurs confirmée par l'application d'une procédure de soustraction de spectre effectuée en retranchant le spectre de la standard $\varepsilon$ Vir (G8III) de celui de HD 174016-7 : le spectre obtenu (Fig. 4) présente de nombreuses similitudes avec celui de l'étoile A0p HD 71866 (Bertaud \& Floquet 1974).

C'est, à notre connaissance, après HD 59435 (Wade et al. 1996), la seconde étoile Ap trouvée dans une étoile à spectre composite : ce fait mérite donc d'être souligné ! On peut noter aussi que le spectre IR ne nous avait donné aucun soupçon sur le caractère Ap de cette composante contrairement aux cas des étoiles secondaires de type Am. 
Confrontation aux données d'Hipparcos Le catalogue HIPPARCOS (1997) donne pour le système : $V=7,44 ; \pi=2,10 \pm 0,57$ (soit une distance d'environ 480 parsecs) ; $B-V=0,455 \pm 0,015$. Nous en déduisons que la magnitude absolue visuelle globale du couple est : $-0,43<M v<-1,64 \mathrm{mag}$, ces bornes correspondant à une erreur de $\pm 1 \sigma$ sur la parallaxe. Ces divers éléments nous amènent à proposer pour HD 174016-7 le modèle résumé dans le tableau ci-dessous :

\begin{tabular}{ccccc}
\hline & Primaire & Secondaire & $\begin{array}{c}\text { Système } \\
\text { (modèle) }\end{array}$ & $\begin{array}{c}\text { Système } \\
\text { (observé) }\end{array}$ \\
\hline Spectre & G6III & A0Vp & & G6III+A0p \\
$M_{v}$ & 0 & 0,6 & $-0,5$ & {$[-1,6 ;-0,4]$} \\
$B-V$ & 0,88 & $-0,02$ & 0,46 & 0,455 \\
\hline
\end{tabular}

dans lequel les indices de couleur figurant en Cols. 2, 3 et 4 correspondent aux calibrations de Schmidt-Kaler (1982). Ces paramètres, compte tenu des spectres observés, sont ceux qui satisfont au mieux les données d'HIPPARCOS, $M_{v}$ et $B-V$ globaux du couple, en négligeant l'absorption interstellaire.

\section{Discussion}

\subsection{Masses et paramètres géométriques}

Comme nous venons de le voir, HD 174016-7 est une binaire à spectre composite dont les deux composantes sont détectables avec CORAVEL, ce qui nous permet d'obtenir avec une bonne précision le rapport des masses des composantes, soit $q=K_{1} / K_{2}=0,855 \pm 0,009$. Ce fait, relativement peu fréquent dans les systèmes de ce type (quand la composante chaude est une étoile normale, elle ne donne pas de trace de corrélation à CORAVEL), nous permet ici de faire une estimation assez fiable de la masse de l'étoile géante. Il est en effet établi que les masses des étoiles naines sont bien mieux connues que celles des géantes, qui perdent une part conséquente de leur masse durant leur évolution. Pour la masse de la composante chaude, que nous supposons être celle d'une étoile naine de type A0, nous avons utilisé la relation $\log M /(B-V)_{0}$ donnée par J. Andersen (1991). Dans cet article, Andersen ré-examine la question des masses des étoiles de la séquence principale à la lumière d'un échantillon, soigneusement sélectionné, de 45 systèmes à éclipses. Vu la dispersion des points sur le graphique, nous pouvons estimer à $2,4 \pm 0,1 M_{\odot}$ la masse de la secondaire, ce qui donne pour la primaire géante $M_{1}=2,81 \pm 0,12 M_{\odot}$, compte tenu de la précision de la valeur de $q$. L'échantillon utilisé par Andersen comporte une seule étoile géante, la primaire du système TZ For = HD 20301, de type G8 III (la secondaire étant de type F7 IV). Pour cette étoile, la masse est de $2,05 \pm 0,06 M_{\odot}$, valeur sensiblement plus faible que celle que nous trouvons plus haut. Cette différence peut s'expliquer si l'on considère que la primaire de HD 174016-7, associée à une secondaire naine chaude $\mathrm{A} 0$, est en fait une géante jeune dont la masse doit être proche de celle qu'elle avait quand elle appartenait encore à la séquence principale. Toujours en nous basant sur la valeur présumée de la composante naine chaude, $2,4 M_{\odot}$, nous obtenons $\sin i=0,888$, soit $i=62,6^{\circ}$, et pour séparation moyenne des composantes : $a=a_{1}+a_{2}=1077,7 \mathrm{Gm} \sim 7,2 \mathrm{UA}$. Etant donné la distance du système (476 pc), cette séparation se traduirait par un écart angulaire de 15 millisecondes d'arc (mas), donc au maximum $a(1+e)=24$ mas. Ce résultat est cohérent avec le fait que Hartkopf \& McAlister (1984) n'aient pu séparer les composantes de HD 174016-7 par interférométrie des tavelures, avec un télescope de $4 \mathrm{~m}$ de diamètre permettant seulement une résolution limite de 30 mas.

\subsection{Rotation}

Le profil des traces de corrélation à CORAVEL permet d'accéder à la vitesse de rotation projetée $v \sin i$ (Benz \& Mayor 1981 ; De Medeiros \& Mayor 1999). Cette analyse, effectuée à l'observatoire de Genève conjointement à l'obtention des VR définitives, conduit à $v \sin i=$ $5,1 \pm 0,4 \mathrm{~km} \mathrm{~s}^{-1}$, valeur moyenne obtenue pour la primaire. Cette quantité correspondrait, dans l'hypothèse de coplanarité entre plan équatorial et plan orbital, à une vitesse équatoriale de $5,7 \mathrm{~km} \mathrm{~s}^{-1}$; elle apparaît relativement faible pour une étoile géante mais est néanmoins très audessus de la valeur de synchronisme $\left(\sim 0,2 \mathrm{~km} \mathrm{~s}^{-1}\right)$, ce à quoi on pouvait s'attendre au vu des dimensions du système. Pour la secondaire, le pic de corrélation trop étroit ne permettait pas une estimation valable du $v \sin i$ de cette composante.

\section{3. État d'évolution du système}

Sur le diagramme $M_{\mathrm{bol}} / \log T_{\text {eff }}$ issu du code d'évolution stellaire de Schaller et al. (1992), diagramme qui illustre les trajets évolutifs d'étoiles de différentes masses pour la métallicité solaire, nous avons situé les composantes de HD 174016-7 (Fig. 5). Les points représentatifs de la primaire et de la secondaire du système ont été reportés en fonction des paramètres intrinsèques proposés plus haut : types spectraux, classes de luminosité et magnitudes absolues visuelles. Nous constatons que les masses théoriques données par le diagramme, voisines de $3 M_{\odot}$ pour la primaire et de $2,5 M_{\odot}$ pour la secondaire, sont en très bon accord avec les estimation faites précédemment, ce qui contribue à valider le modèle proposé. La Fig. 5 fournit également des informations sur l'état présumé d'évolution du système : la composante chaude serait environ au milieu de sa durée de vie sur la séquence principale, tandis que la composante froide serait au tout début de son évolution dans la phase d'étoile géante.

Nous conclurons en notant que si nos études dans le bleu et dans l'IR nous ont permis de déceler jusqu'ici un 
certain nombre d'étoiles à raies métalliques (étoiles Am) comme composantes secondaires d'étoiles à spectre composite, avec HD 174016-7 nous trouvons un second cas de SC avec une composante chaude Ap.

Ces associations d'une étoile géante froide et d'une étoile chaude chimiquement particulière n'ont été mises en évidence que récemment et constituent une information importante quant à la connaissance de cette famille de binaires.

\section{Bibliographie}

Abt H.A., 1984, ApJ 285, 247

Andersen J., 1991, A\&AR 3, 91

Baranne A., Mayor M., Poncet J.L., 1979, Vistas Astron. 23, 279

Benz W., Mayor M., 1981, A\&A 93, 235

Bertaud Ch., Floquet M., 1974, A\&AS 16, 71

Cannon A.J., Pickering E.C., 1922, Annals Astron. Obs. Harvard, 97

Carquillat J.M., Ginestet N., Pédoussaut A., 1988, A\&AS 75, 305

Carquillat J.M., Ginestet N., Duquennoy A., Pédoussaut A., 1994, A\&AS 106, 597

De Medeiros J.R., Mayor M., 1999, A\&A (sous presse)
Duquennoy A., 1987, A\&A 178, 114

Ginestet N., Carquillat J.M., Pédoussaut A., 1991, A\&AS 91, 265

Ginestet N., Carquillat J.M., Jaschek C., Jaschek M., 1997, A\&AS 123, 135

Ginestet N., Carquillat J.M., Jaschek C., 1999, A\&AS 134, 473

Griffin R.F., 1967, ApJ 148, 465

Griffin R.\&R., 1986, JA\&A 7, 195

Griffin R.\&R., 1988, Observatory 108, 114

Griffin R.F., Carquillat J.M., Ginestet N., Duquennoy A., Udry S., 1997, Observatory 117, 351

Hartkopf W.I., McAlister H.A., 1984, PASP 96, 105

Hauck B., 1986, A\&AS 64, 21

The HIPPARCOS and TYCHO Catalogues, ESA, Noordwijk, (ESA SP-1200), 1997

Hynek J.A., 1938, Contr. Perkins Obs. 1, 185

Nadal R., Ginestet N., Carquillat J.M., Pédoussaut A., 1979, A\&AS 35, 203

Olsen E.H., 1980, A\&AS 39, 205

Schaller G., Schaerer D., Meynet G., Maeder A., 1992, A\&AS 96, 269

Schmidt-Kaler Th., 1982, Landolt-Börnstein, New series, Gr. 6, Vol. 2, Subvol. B. Springer-Verlag; Berlin, Heidelberg, New-York

Wade G.A., North P., Mathys G., Hubrig S., 1996, A\&A 314, 491 